\title{
Effects of the Olive Tree Leaf Constituents on Myocardial Oxidative Damage and Atherosclerosis
}

Authors

Affiliations
Panagiotis Efentakis ${ }^{1}$, Efstathios K. Iliodromitis ${ }^{2}$, Emmanuel Mikros ${ }^{1}$, Anastasia Papachristodoulou ${ }^{1}$, Nikolaos Dagres ${ }^{2}$, Alexios-Leandros Skaltsounis ${ }^{3}$, loanna Andreadou

${ }^{1}$ Department of Pharmaceutical Chemistry, Faculty of Pharmacy, University of Athens, Athens, Greece

2 Second Department of Cardiology, University of Athens Medical School, Attikon University Hospital, Athens, Greece

${ }^{3}$ Laboratory of Pharmacognocy and Chemistry of Natural Products, Faculty of Pharmacy, University of Athens, Athens, Greece

\section{Key words}

- olive leaf constituents

- oxidative stress

- LDL oxidation

- anti-atherosclerotic effects

- anti-ischemic effects

- Olea europaea

- Oleaceae received August 4, 2014

revised April 6, 2015

accepted April 7, 2015

Bibliography

DOI http://dx.doi.org/

10.1055/s-0035-1546017

Published online May 27, 2015

Planta Med 2015; 81: 648-654

(c) Georg Thieme Verlag KG

Stuttgart · New York .

ISSN 0032-0943

\section{Correspondence}

Dr. loanna Andreadou

Department of Pharmaceutical Chemistry

University of Athens School of Pharmacy

Panepistimiopolis, Zografou

Athens 15771

Greece

Phone: + 302107274827

Fax: + 302107274747

jandread@pharm.uoa.gr

\section{Abstract}

The olive (Olea europaea) leaf is considered an important traditional herbal medicine utilized against infectious diseases, and for the treatment of diabetes and hypertension. Moreover, olive leaf constituents have been related to cardioprotection, probably due to their association with cellular redox modulating effects. The pathogenesis of certain common diseases, including those of the cardiovascular system, involves oxidative stress

\section{Introduction}

$\nabla$

The beneficial effects of olive leaf extract are known since antiquity, with numerous records confirming its therapeutic use [1]. During the last two centuries, there has been increasing interest in investigating the pharmacological properties of the constituents of olive leaves, and it has been shown that they can exhibit a variety of biological actions, such as antioxidant [2,3], antimicrobial [3], antihypertensive [4-6], vasodilator [7], and hypoglycemic [8] properties. Clinical data on olive leaf extract are apparent since 1950 [1], with phenolic extract exhibiting the most promising beneficial effect. The wide use of oil, olives, and leaf extracts by the Mediterranean population for the alleviation of inflammatory diseases, gout, and blood pressure led to the incorporation of the $80 \%$ ethanolic olive leaf dry extract (OLE) in the European Pharmacopoeia (Ph. Eur.) [9]. According to the Ph. Eur., the most abundant substances in standardized dry leaf extracts are oleuropein, hydroxytyrosol, caffeic acid, tyrosol, apigenin, and verbascoside, with oleuropein being the major component of olive leaf extract [10].

Enzymatic hydrolysis of oleuropein leads to the formation of oleuropein aglycone, which is further hydrolyzed to hydroxytyrosol (also known as 3,4-dihydroxyphenylethanol or 3,4-DHPEA), and tissue inflammation. Olive polyphenolic compounds, such as oleuropein, hydroxytyrosol, or tyrosol, possess antioxidant, anti-inflammatory, antiatherosclerotic, anti-ischemic, and hypolipidemic effects on the myocardium as demonstrated by various in vitro and in vivo studies. In this review article, we summarize the current knowledge on the role of the olive leaf constituents in the prevention of cardiac dysfunction and highlight future perspectives in their use as cardioprotective agents in therapeutics. and other non-glycosidic secoiridoids (i.e., elenolic acid). Chemical hydrolysis of ligstroside, which has also been isolated from 0 . europaea L. leaf extract leads to the formation of tyrosol (also known as 4-hydroxyphenylethanol) [11]. Another derivative of oleuropein metabolic degradation is oleacein (also referred to as 3,4-DHPEA-EDA or oleuropein aglycone decarboxymethyldialdehyde form or noroleuropein aglycone).

The polyphenols that are present in olive leaves have been shown to be potent antioxidant and radical scavengers, exhibiting many therapeutic properties such as antitumor and anti-inflammatory ones [12]. Furthermore, the effects of olive polyphenols on scavenging lipid peroxyl radicals within membranes were associated with their beneficial effects for human skin protection [13]. In general, olive constituents exhibit a wide range of beneficial properties via antioxidant modulation in the management of diseases in which neuroprotective, gastroprotective, antidiabetic, or anti-ageing activities may play a beneficial role (reviewed in [14]).

Plant-derived compounds represent potential sources for molecules that may be used for the development of new drugs especially designed for the treatment and/or control of chronic inflammatory states, such as atherosclerosis and thrombogenicity [15], related to cardiovascular diseases 
(CVDs). The increasing number of patients around the world suffering from CVD indicates the need for innovative strategies for more effective CVD prevention and treatment [16-18]. In this review article, we summarize the current knowledge of the role of the major olive leaf constituents, oleuropein and its metabolites oleuropein aglycone, hydroxytyrosol, oleacein, elenolic acid as well as tyrosol, on the myocardium and highlight future perspectives of their use as cardioprotective agents. The above compounds have been selected as their presence in olives has been related to the unique bioactivity of olive-related foods, unlike constituents such as caffeic acid and apigenin, which are abundant in a wide range of plant extracts.

\section{Myocardial Oxidative Stress}

$\nabla$

The damage of the cardiovascular endothelium is one of the first steps in the path to heart disease. The endothelium is a direct target of all of the major risk factors for heart disease such as diabetes, hyperlipidemia, hyperglycemia, inflammation, ageing, and hypertension. The common element in all of these pathophysiological conditions is the formation of reactive oxygen species (ROS) [19]. Many data have demonstrated that ROS production by endothelial mitochondria contributes to heart disease (reviewed in [20]). Moreover, oxidative stress within ventricular myocytes can also be detrimental to the heart. In fact, much of the contractile dysfunction and adverse myocardial remodeling, which has been observed in a wide range of cardiomyopathies, involves oxidative stress and endothelial nitric oxide synthase (eNOS) uncoupling leading to a decrease in NO production and an increase in ROS formation [21-23].

However, the oxidative damage occurs only when the oxidative stress levels exceed the antioxidant defense capacity. As already shown in patients with CVD, the activity of the endogenous antioxidant enzymes is reduced [24]. The manifestation and progression of CVDs are concomitant with a downregulation of enzymes such as superoxide dismutase (SOD), glutathionine peroxidase (GPx), and catalase and proteins such as albumin and metallothionein, which are known to exhibit antioxidant protection in the myocardium [25]. Thus, the heart is one of the most susceptible organs to free radical-mediated oxidative stress [26]. Therefore, scavenging of ROS may have various protective and beneficial effects on the heart.

\section{Atherosclerosis}

$\nabla$

Atherosclerosis is the primary cause of CVD and the underlying cause of more than $50 \%$ of all deaths in Westernized societies. CVD is considered to be a consequence of atherogenesis and is a result of a complex network of interactions between several risk factors and endogenous cell signaling between arterial wall cells. It is well established that dyslipidemia, diabetes, and platelet aggregation can lead to an impairment of the endothelial function and the formation of atheroma [27]. Atherosclerosis is a multifactorial disease in which inflammation and oxidative stress play an important role. Inflammatory and immunological responses contribute to the formation and rupture of atherosclerotic plaques and occlusion of the coronary artery $[28,29]$. The formation of ROS can be mediated through the activity of enzymes, such as NADH/NADPH and xanthine oxidase [30], reactions with products of the mitochondrial electron transport chain [31], and un- coupling of eNOS [32-34]. The oxidation of low-density lipoproteins (LDL) by ROS is a prominent factor in the formation of atheroma in the beginning of the evolution of the disease [35]. Endothelial dysfunction is connected to the presence of oxidized LDL (ox-LDL), which leads to a signaling cascade that includes protein expression inducing atherogenesis and heart disease [36,37]. Furthermore, the aggregation and degranulation of platelets seem to trigger the formation of thrombus and coronary artery occlusion, with platelet activation factor (PAF) being a critical factor for platelet aggregation [38].

\section{Oleuropein}

$\nabla$

Myocardial oxidative stress and low-density lipoprotein oxidation

Oleuropein has been shown to possess high antioxidant activity in vitro when it is compared to a water-soluble analog of tocopherol [39]. Furthermore, oleuropein scavenges superoxide anions and hydroxyl radicals, and inhibits the respiratory burst of neutrophils and hypochlorous acid-derived radicals [39-41]. In vitro studies have shown that oleuropein significantly reduced vitamin E consumption in an LDL-oxidation model, and it has been claimed that this component possesses high antioxidant properties [42]. In isolated rat hearts, significant protection against ischemia/reperfusion-induced oxidative stress was observed by oleuropein administration at a dose comparable to the average daily intake of biophenols from olive oil in the Mediterranean diet [43].

Furthermore, our group has shown that chronic treatment with oleuropein at a dose that was based on the average consumption of olive drupes and olive oil in the Mediterranean area significantly reduced circulatory oxidative stress biomarkers, such as malondialdehyde (MDA) and protein carbonyls, and enhanced SOD activity in normal and atheromatic rabbits that were subjected to ischemia/reperfusion [44]. We have also shown that oleuropein at a nutraceutical dose successfully treated doxorubicin (DXR)-induced cardiomyopathy in in vivo rat models by decreasing the oxidative stress biomarkers and reducing inducible nitric oxide synthase (iNOS) in cardiomyocytes $[45,46]$, with a parallel restoration of the unbalanced metabolite profile in the myocardium [47].

The outcome of the Masella et al. study showed that oleuropein completely prevented the murine J774 A.1 macrophage-like cellmediated oxidation of LDL along with a reduction of oxygen species production while restoring the expression of glutathione-associated proteins [48]. The addition of $10 \%(\mathrm{w} / \mathrm{w})$ extra virgin olive oil and $7 \mathrm{mg} / \mathrm{kg}$ oleuropein to the standard diet in rabbits increased the ability of LDL to resist oxidation [49].

\section{Anti-atherosclerotic and anti-ischemic effects}

In vitro results verify that various compounds originating from olives exhibit an antithrombotic effect with different potencies by inhibiting the phosphodiesterase (PDE) signaling pathway, whereas oleuropein was found to present the highest activity among phenols and flavonoids [50]. In addition, oleuropein exhibited the greatest potency as an anti-atherosclerotic agent among the other phenolic constituents originating from the olive tree [5].

One study has been performed in isolated rat hearts to investigate the direct cardioprotective effect of oleuropein in acute events that follow coronary occlusion. Pretreatment with oleuro- 
pein before ischemia resulted in a significant decrease in creatine kinase and reduced glutathione release in the perfusate, likely because of its antioxidant properties [43]. We have also shown that oleuropein reduced the infarct size in normal rabbits and, at higher doses, in hypercholesterolemic rabbits in vivo. Furthermore, it reduced total cholesterol and triglyceride levels, altered the metabolic profile, and provided cardioprotection even before the onset of ischemia [44].

\section{Oleuropein Aglycone \\ $\nabla$}

Myocardial oxidative stress and

low-density lipoprotein oxidation

Other 0 . europaea metabolites also present beneficial actions. Oleuropein aglycone, also referred to as 3,4-DHPEA-EA, shows antioxidant activity comparable with that of caffeic acid, oleuropein, and hydroxytyrosol. An in vitro study evaluating the effect of oleuropein aglycone on the modification of Cu-stimulated human LDL revealed that this particular compound reduced the kinetic and extent of lipid peroxidation [51].

\section{Hydroxytyrosol \\ $\nabla$}

Myocardial oxidative stress and

low-density lipoprotein oxidation

The polyphenol hydroxytyrosol has been found to play a protective role against cardiovascular diseases and is considered a potent antioxidant factor [52]. In vitro studies on human neutrophils showed that this biophenol can eliminate $\mathrm{N}$-formyl-methionyl-leucylphenylalanine (fMLP), phorbol myristate acetate (PMA), and opsonized zymosan-induced damage, which is mediated by hydrogen peroxide under oxidative conditions [53]. However, hydroxytyrosol had no effect on lucigenin-amplified chemiluminescence, suggesting that it does not inhibit NADPH oxidase activation or scavenge superoxide anions [53]. Additionally, experiments evaluating the cytotoxicity, apoptosis, and cell cycle-related effects of hydroxytyrosol in various cell lines showed that it causes an upregulation of numerous antioxidant proteins and enzymes, including heme-oxygenase-1, glutaredoxin, and glutathione peroxidase [54]. The antioxidant activity of hydroxytyrosol on $\mathrm{H}_{2} \mathrm{O}_{2}$-induced intracellular $\mathrm{ROS}$ has been demonstrated in porcine pulmonary artery endothelial cells (VECs). Herein, hydroxytyrosol showed its efficacy to regulate the antioxidant defense system in VECs by inducing the phosphorylation of 5'-adenosine monophosphate-activated protein kinase (AMPK) with subsequent activation of Forkhead box 03 (FOXO3a) and catalase expression [55]. Furthermore, it has also been shown to improve cardiac disturbances induced by DXR by significantly reducing the percentage of injured mitochondria and oxidative damage, leading to the conclusion that hydroxytyrosol improved the mitochondrial electron transport chain leading to cell survival [56].

Hydroxytyrosol also seems to be a potent inhibitor of LDL oxidation, as it inhibits the production of isoprostanes, which are formed during LDL oxidation [57].

\section{Anti-atherosclerotic and anti-ischemic effects}

Although the antioxidant properties of hydroxytyrosol have been confirmed in both in vivo and in vitro studies, data on the antiatherosclerotic activity of hydroxytyrosol are less promising. Hy- percholesterolemia decreases NO bioavailability via the downregulation of eNOS in association with an increased production of oxygen-derived free radicals that may inactivate NO, thus the upregulation of eNOS contributes to the protection against atherosclerosis [58]. An in vitro study on human endothelial cells, which correlated eNOS upregulation with hydroxytyrosol protective activity, concluded that the treatment of cells with a wide range of hydroxytyrosol concentrations did not exhibit positive results as far eNOS and NO bioavailability are concerned. This leads to the conclusion that the anti-atherosclerotic properties of this compound are not eNOS mediated [59]. However, another in vitro study showed that hydroxytyrosol exhibits anti-atherosclerotic properties by reducing monocytoid cell adhesion to stimulated endothelium, as well as reducing the vascular cell adhesion molecule-1 (VCAM-1) [60].

The results originating from in vivo models are also divergent. The intake of hydroxytyrosol in an in vivo apo-E knockout mice model showed that this compound, when administered for 10 weeks, led to atherosclerotic lesions associated with the activation of monocytes and modification of the blood lipid profile. The study claimed that under certain conditions the phenol could be rather harmful than cytoprotective [61]. However, another animal study showed that a one-month intake of hydroxytyrosol by rabbits consuming an atherogenic diet resulted in an improved antioxidant capacity of the myocardium and a reduced size of atherosclerotic lesions compared to control animals [52]. The anti-ischemic properties of hydroxytyrosol have been proven for other tissues and organs apart from myocardium. The administration of hydroxytyrosol in mice protected hepatic cells from ischemia/reperfusion injury by inducing a parallel decrease in oxidative stress levels [62].

\section{Elenolic Acid}

Elenolic acid did not possess any significant effects on lipopolysacharide (LPS)-stimulated expression of the adhesion molecule VCAM-1 in HUVECs and bovine aortic endothelial cells (BAECs) [60], indicating that the cardioprotective effects of elenolic acid are not evident. However, studies on its effect on the myocardium are scarce.

\section{Oleacein}

$\nabla$

Myocardial oxidative stress and

low-density lipoprotein oxidation

One of the most important derivatives of oleuropein metabolic degradation is oleacein. It has been shown to exhibit improved antioxidant properties compared to oleuropein [63]. It has been reported that the antioxidant activity of virgin olive oil is principally due to oleacein [64]. Oleacein exhibited anti-inflammatory activity on monocytes by significantly inhibiting the production of ROS and efficiently reducing cycloxygenase-2 (COX-2) mRNA synthesis. These findings show an ability of oleacein to modulate proinflammatory molecule production [65]. Another in vitro study revealed that oleacein could permeate the cell membrane, thus exhibiting antioxidant activity inside the red blood cells (RBCs), fortifying the antioxidant capacity of the circulating blood [66]. The protective role of oleacein on erythrocytes was also proven by another group of investigators, who observed that it significantly protected RBCs from oxidative hemolysis induced by 
$\mathrm{H}_{2} \mathrm{O}_{2}$, a protection motif exhibited by nearly all phenolic components found in olive extracts [67].

\section{Anti-atherosclerotic and anti-ischemic effects}

Oleacein has been proven to directly inhibit 5-lipoxygenase [68]. Moreover, oleacein inhibited neutral endopeptidase (NEP) activity, elastase, metalloproteinase-9 (MMP-9), and interleukin-8 (IL8) release from neutrophils, thus protecting the endothelium from pathogenic alterations [69]. The protective actions of oleacein on the endothelium were also demonstrated by the fact that the pretreatment of cells with oleacein resulted in a dose-dependent inhibition of the proinflammatory $\mathrm{CCl} 2$ chemokine production and monocyte adhesion to human umbilical vein endothelial cells (HUVECs) [70].

However, to the best of our knowledge, there is no study, so far, investigating the anti-ischemic effects of oleacein.

\section{Tyrosol \\ $\nabla$}

\section{Myocardial oxidative stress and} low-density lipoprotein oxidation

Tyrosol has been found to reduce lipid peroxidation products in cells, but to a significantly lesser extent than hydroxytyrosol, probably because of its lower scavenger ability. However, while accumulated intracellularly, it could preserve an antioxidant defense in cells [71]. Another in vitro study led to the conclusion that tyrosol strengthened the resistance to oxidative stress in the nematode Caenorhabditis elegans and extended its lifespan. This evidence was supported by proteomic studies identifying protein levels that were differentially expressed in nematodes grown in a medium containing tyrosol [72].

A study on macrophages found that tyrosol inhibited $\mathrm{H}_{2} \mathrm{O}_{2}$ production induced by ox-LDL as well as arachidonic acid release and prostaglandin E2 (PGE2) synthesis. However, these effects of tyrosol were not mediated through interference of the compound with ox-LDL receptors, indicating that tyrosol abrogates the deleterious effects of ox-LDL [73]. Tyrosol possesses a binding capacity on LDL as exhibited by in vitro data and LDL composition is modified by one week administration of this phenolic compound through diet [74]. An antioxidant capacity of tyrosol has been evaluated neither in vivo nor ex vivo.

\section{Anti-atherosclerotic and anti-ischemic effects}

It has been shown in vitro that tyrosol could activate a molecular pathway, which includes phosphorylation of protein kinase $B$ (PKB/Akt), expression of sirtuin 1 gene (SIRT1), and, as a result, the deactivation of transcription factor FOXO-3a activity, which is known to mediate apoptosis in cells. An additional element to the above results was the upregulation of eNOS, which contributes to the cardioprotective activity of tyrosol [75]. In an ex vivo study on Laggendorf perfused rat hearts, tyrosol was able to diminish infarct size when administered $30 \mathrm{~min}$ before the incidence of sustained ischemia. The underlying mechanism of the above cardioprotective effect of tyrosol was attributed to the activation of Akt and eNOS [76]. Experiments on H9c2 cardiomyocytes showed that tyrosol can ameliorate ischemia-reperfusion injury via inhibition of nuclear condensation, reduced caspase-3 activity, cytochrome c release, and c-Jun $\mathrm{N}$-terminal kinases (JNK) activation [77].

\section{Comparative and Synergistic Activities of Olive Leaf Extract Constituents \\ $\nabla$}

Myocardial oxidative damage

Oleuropein and hydroxytyrosol exhibited the greatest potency as anti-atherosclerotic agents among the other phenolic constituents originating from the olive tree. However, hydroxytyrosol possessed a lower $\mathrm{EC}_{50}$ than oleuropein, indicating that this certain constituent is a better free-radical scavenger and possesses an improved protective factor against LDL oxidation [5]. A recent study compared the protective effect of the ethanolic and methanolic extracts of olive leaves with the effects of oleuropein, hydroxytyrosol, and quercetin as positive standards in a carbonyl compound (4-hydroxynonenal)-induced model of oxidative damage to rat cardiomyocytes (H9c2). The ethanolic extract of olive leaves, which contains larger amounts of oleuropein and hydroxytyrosol than the methanolic one, showed better protection on cardiomyocyte viability than the methanolic extract or each isolated phenolic compound against 4-hydroxynonenal-induced oxidative stress and toxicity [78]. Another study compared the capacity of four important olive polyphenolic compounds (oleuropein, hydroxytyrosol, 3,4-DHPEA-EA, and 3,4-DHPEA-EDA) to protect red blood cells (RBCs) from oxidative hemolysis induced by $\mathrm{H}_{2} \mathrm{O}_{2}$. All compounds revealed significant protection to RBCs from oxidative hemolysis induced by $\mathrm{H}_{2} \mathrm{O}_{2}$ with the order of activity being:

oleacein > oleuropein-aglycone > hydroxytyrosol=oleuropein [79].

Treatment of the isolated rat aorta in ex vivo studies with oleuropein, tyrosol, and hydroxytyrosol showed that only hydroxytyrosol could provide protection against cumene hydroperoxide (CHP)-induced damage in the NO-mediated relaxation when CHP was used to stimulate oxidative stress [80]. Hydroxytyrosol exhibited the highest antioxidant activity compared to oleuropein and tyrosol in ex vivo experiments on the rat aorta [80].

Protection against oxidative stress induced by oleacein and other metabolites originating from olive leaves extract is also evident. Human peripheral blood mononuclear cells (PBMC) and promyelocytic leukemia cells (HL60) treated with hydroxytyrosol as well as 3,4-DHPEA-EA, oleuropein, and tyrosol, [p-hydroxyphenylethanol (p-HPEA)] the dialdehydic form of elenolic acid linked to tyrosol, appeared to be protective against DNA damage [81]. Endothelial progenitors cells (EPCs) are responsible for neovascularization of ischemic tissue and may participate in the de novo formation of endothelium on an injured arterial wall. Oleacein along with oleuropein pretreatment of EPCs increased cell survival and telomerase activity, and decreased the percentage of senescent cells and intracellular ROS production. This effect was related to NF-E2-related factor 2 (Nrf2) transcription factor activation and to the increase of heme-oxygenase-1 (HO-1) expression [82].

Olive phenols are correlated with antioxidant protection against ageing-induced damage. The administration of biophenols originating from olives could retain the rise of oxidative stress biomarkers in the heart, such as protein carbonyls, and raise the levels of Nrf2 and Nrf2-associated gene expression, indicating that the olive polyphenolic fraction is a promising factor of protection against ageing-induced oxidative stress [83]. Furthermore, when olive polyphenols are added to isolate LDL and treated with various oxidative agents, they achieve a significant reduction of lipoprotein oxidation in vitro [84]. These results indicate that olive oil phenolic compounds protect LDL against peroxyl radical-de- 
pendent and metal-induced oxidation and could associate with LDL after their incubation with plasma [84].

\section{Anti-atherosclerotic and anti-ischemic effects}

Olive leaf extract treatment attenuated the metabolic, structural, and functional changes in the heart in rats with a diet-induced metabolic syndrome through the antioxidant and anti-inflammatory effects of polyphenols, mainly oleuropein and hydroxytyrosol [85]. Olive leaf extract reduced serum levels of triglycerides, total cholesterol, LDL, HDL, and MDA in atherosclerotic rabbits with a parallel downregulation of monocyte chemoattractant protein-1 (MCP-1), VCAM-1, nuclear factor kappa-lightchain-enhancer of activated B cells (NF-kB) and tumor necrosis factor- $\alpha$ (TNF- $\alpha$ ) [86]. Moreover, olive leaf extract was found to reduce the formation of conjugated dienes and thiobarbituric acid reactive substances (TBARS), protecting LDL against oxidation in vitro, deducing that it is a source of potent antioxidants and anti-atherosclerotic factors [87].

A clinical study was conducted in order to determine the effects of 0 . europaea $L$. leavf extract on platelet function in healthy human subjects. The product was stated to contain $5.40 \mathrm{mg} / \mathrm{mL}$ of oleuropein, which was considered its active ingredient. The results of the study clarified that oleuropein diminished the increased inflammation markers, mediated through phospholipase $\mathrm{C}$ and arachidonic acid metabolism, and reduced the elevated hydrogen peroxide concentrations together with platelet activation [88].

\section{Conclusion}

In this review we provided an overview of the recent advances and current knowledge on olive leaf constituents, namely oleuropein, oleuropein aglycone, hydroxytyrosol, oleacein, elenolic acid, and tyrosol, on myocardial oxidative stress and atherosclerosis.

It has been proven that phenolic compounds of olive leaf extracts are capable of reducing oxidative stress levels in animal experimental models. This effect is mainly ascribed to hydroxytyrosol and oleuropein. Defense against LDL oxidation is also induced by olive components in vitro, while a concomitant increase in glutathionine-assosiated enzymes and hypolipidemic effects of olive derivatives has been observed in vivo. Although among the phenolic compounds, hydroxytyrosol, tyrosol, and oleuropein are endowed with significant antioxidant properties, the anti-atherosclerotic, hypocholesterolemic, and anti-ischemic activities of the above compounds have not been studied in detail. Protection against ischemia reperfusion is evident during chronic administration of oleuropein before the ischemic episode in an in vivo model. Findings concerning hydroxytyrosol are not that preferable, considering that the administration of the biophenol in an in vivo model led to an increased atherosclerotic incidence. Additionally, studies concerning the cytoprotective effects of hydroxytyrosol against ischemia-reperfusion injury are scarce.

In conclusion, oleuropein seems to be a promising molecule that may be used as a cardioprotective agent. However, the underlying signaling cascades of its cardioprotective effects remain to be elucidated.

Conclusively, O. europaea L. leaf constituents possess proven beneficial results on myocardial oxidative stress and atherosclerosis. Basic research is mandatory in order to investigate olive leaf constituents as pharmacological tools for the prevention and protec- tion of heart diseases. Furthermore, there is a further need for research concerning the outcomes and clinical trials that have been carried out in order to obtain solid evidence for these claims that could be translated in clinical practice.

\section{Conflict of Interest}

$\nabla$

None.

\section{References}

1 Bartolini G, Petruccelli R. Classification, origin, diffusion and history of the olive. Rome: Food and Agriculture Organisation of the United Nations; 2002

2 Le Tutour B, Guedon D. Antioxidative activities of Olea europaea leaves and related phenolic compounds. Phytochemistry 1992; 31: 11731178

3 Ghisalberti EL. Biological and pharmacological activity of naturally occurring iridoids and secoiridoids. Phytomedicine 1998; 5: 147-163

4 Khayyal MT, el-Ghazaly MA, Abdallah DM, Nassar NN, Okpanyi SN, Kreuter $M H$. Blood pressure lowering effect of an olive leaf extract (Olea europaea) in L-NAME induced hypertension in rats. Arzneimittelforschung 2002; 52: 797-802

5 Visioli F, Galli C. The effect of minor constituents of olive oil on cardiovascular disease: new findings. Nutr Rev 1998; 56: 142-147

6 Jänicke C, Grünwald J, Brendler T. Handbuch Phytotherapie. Stuttgart: Wissenschaftliche Verlagsgesellschaft; 2003

7 Zarzuelo A, Duarte J, Jimenez J, Gonzalez M, Utrilla MP. Vasodilator effect of olive leaf. Planta Med 1991; 57: 417-419

8 Gonzalez M, Zarzuelo A, Gamez MJ, Utrilla MP, Jimenez J, Osuna I. Hypoglycemic activity of olive leaf. Planta Med 1992; 58: 513-515

9 Flemmig J, Rusch D, Czerwinska ME, Rauwald HW, Arnhold J. Components of a standardised olive leaf dry extract (Ph. Eur.) promote hypothiocyanite production by lactoperoxidase. Arch Biochem Biophys 2014; 549: 17-25

10 Flemmig J, Kuchta K, Arnhold J, Rauwald HW. Olea europaea leaf (Ph. Eur.) extract as well as several of its isolated phenolics inhibit the gout-related enzyme xanthine oxidase. Phytomedicine 2011; 18: 561-566

11 Briante R, Patumi M, Terenziani S, Bismuto E, Febbraio F, Nucci R. Olea europaea L. leaf extract and derivatives: antioxidant properties. J Agric Food Chem 2002; 50: 4934-4940

12 Barbaro B, Toietta G, Maggio R, Arciello M, Tarocchi M, Galli A, Balsano C. Effects of the olive-derived polyphenol oleuropein on human health. Int J Mol Sci 2014; 15: 18508-18524

13 Saija A, Uccella N. Olive Biophenols: functional effects on human wellbeing. Trends Food Sci Tech 2001; 11: 357-363

14 Rahmani AH, Albutti AS, Aly SM. Therapeutics role of olive fruits/oil in the prevention of diseases via modulation of anti-oxidant, anti-tumour and genetic activity. Int J Clin Exp Med 2014; 7: 799-808

15 Calixto JB, Campos MM, Otuki MF, Santos AR. Anti-inflammatory compounds of plant origin. Part II. modulation of pro-inflammatory cytokines, chemokines and adhesion molecules. Planta Med 2004; 70: 93103

16 Trichopoulou A, Costacou T, Bamia C, Trichopoulos D. Adherence to a Mediterranean diet and survival in a Greek population. N Engl J Med 2003; 26: 2599-2608

17 Sofi F, Abbate R, Gensini GF, Casini A. Accruing evidence on benefits of adherence to the Mediterranean diet on health: an updated systematic review and meta-analysis. Am J Clin Nutr 2010; 92: 1189-1196

18 Roman B, Carta L, Martínez-González MA, Serra-Majem L. Effectiveness of the Mediterranean diet in the elderly. Clin Interv Aging 2008; 3: 97109

19 Davidson SM, Duchen MR. Endothelial mitochondria: contributing to vascular function and disease. Circ Res 2007; 100: 1128-1141

20 Davidson SM. Endothelial mitochondria and heart disease. Cardiovasc Res 2010; 88: 58-66

21 Manson JE, Greenland P, LaCroix AZ, Stefanick ML, Mouton CP, Oberman A, Perri MG, Sheps DS, Pettinger MB, Siscovick DS. Walking compared with vigorous exercise for the prevention of cardiovascular events in women. N Engl J Med 2002; 347: 716-725

22 Calvert JW, Condit ME, Aragón JP, Nicholson CK, Moody BF, Hood RL, Sindler AL, Gundewar S, Seals DR, Barouch LA, Lefer DJ. Exercise protects 
against myocardial ischemia-reperfusion injury via stimulation of $\beta 3$ adrenergic receptors and increased nitric oxide signaling: role of nitrite and nitrosothiols. Circ Res 2011; 108: 1448-1458

23 Roof SR, Ho HT, Little SC, Ostler JE, Brundage EA, Periasamy M, Villamena FA, Gyorke S, Biesiadecki BJ, Heymes C, Houser SR, Davis JP, Ziolo MT. Obligatory role of neuronal nitric oxide synthase in the heart's anti-oxidant adaptation with exercise. J Mol Cell Cardiol 2015; 81: 54-61

24 Landmesser U, Spiekermann S, Dikalov S, Tatge H, Wilke R, Kohler C, Harrison $D G$, Horning $B$, Drexler $H$. Vascular oxidative stress and endothelial dysfunction in patients with chronic heart failure: role of xanthineoxidase and extracellular superoxide dismutase. Circulation 2002; 106: 3073-3078

25 Landmesser U, Merten R, Spiekermann S, Buttner K, Drexler H, Horning B. Vascular endothelial superoxide desmutase activity in patients with coronary artery disease. Relation to endothelium-dependent vasodilation. Circulation 2000; 101: 2264-2270

26 Andreadou I, Iliodromitis EK, Farmakis D, Kremastinos DT. To prevent, protect and save the ischemic heart: antioxidants revisited. Expert Opin Ther Targets 2009; 13: 945-956

27 Libby P, Theroux P. Pathophysiology of coronary artery disease. Circulation 2005; 111: 3481-3488

28 Navab M, Berliner JA, Watson AD, Hama SY, Territo MC, Lusis AJ, Shih DM, Van Lenten BJ, Frank JS, Demer LL, Edwards PA, Fogelman AM. The Yin and Yang of oxidation in the development of the fatty streak. A review based on the 1994 George Lyman Duff Memorial Lecture. Arterioscler Thromb Vasc Biol 1996; 16: 831-842

29 Hansson GK, Libby P. The immune response in atherosclerosis: a double-edged sword. Nat Rev Immunol 2006; 6: 508-519

30 Madamanchi NR, Vendrov A, Runge MS. Oxidative stress and vascular disease. Arterioscler Thromb Vasc Biol 2005; 25: 29-38

31 Boveris A, Cadenas E, Stoppani AO. Role of ubiquinone in the mitochondrial generation of hydrogen peroxide. Biochem J 1975; 156: 435-444

32 Laursen JB, Somers M, Kurz S, McCann L, Warnholtz A, Freeman BA, Tarpey $M$, Fukai T, Harrison $D G$. Endothelial regulation of vasomotion in apoE-deficient mice: implications for interactions between peroxynitrite and tetrahydrobiopterin. Circulation 2001; 103: 1282-1288

33 Landmesser U, Dikalov S, Price SR, McCann L, Fukai T, Holland SM, Mitch $W E$, Harrison DG. Oxidation of tetrahydrobiopterin leads to uncoupling of endothelial cell nitric oxide synthase in hypertension. J Clin Invest 2003; 111: 1201-1209

34 Kang JJ, Shu L, Park JL, Shayman JA, Bodary PF. Endothelial nitric oxide synthase uncoupling and microvascular dysfunction in the mesentery of mice deficient in $\alpha$-galactosidase A. Am J Physiol Gastrointest Liver Physiol 2014; 306: G140-G146

35 Young S, McEneny J. Lipoprotein oxidation and atherosclerosis. Biochem Soc Trans 2001; 29: 358-362

36 Li D, Mehta JL. Oxidized LDL, a critical factor in atherogenesis. Cardiovasc Res 2005; 68: 353-354

37 Holvoet P. Oxidized LDL and coronary heart disease. Acta Cardiol 2004; 59: $479-484$

38 Yost CC, Weyrich AS, Zimmerman GA. The platelet activating factor (PAF) signaling cascade in systemic inflammatory responses. Biochimie 2010; 92: 692-697

39 Speroni E, Guerra MC, Minghetti A, Crespi-Perellino N, Pasini P, Piazza F. Oleuropein evaluated in vitro and in vivo as an anti-oxidant. Phytother Res 1998; 12: 98-100

40 Visioli F, Bellomo G, Galli C. Free radical scavenging properties of olive oil polyphenols. Biochem Biophys Res Commun 1998; 247: 60-64

41 Chimi H, Cillard J, Cillard P, Rahmani M. Peroxyl and hydroxyl radical scavenging activity of some natural phenolic anti-oxidants. J Am Oil Chem Soc 1991; 68: 307-312

42 Visioli F, Galli $C$. The effect of minor constituents of olive oil on cardiovascular disease: new findings. Biochem Biophys Res Commun 1998; 56: $142-147$

43 Manna C, Migliardi V, Golino P, Scognamiglio A, Galletti P, Chiariello M, Zappia $V$. Oleuropein prevents oxidative myocardial injury induced by ischemia and reperfusion. J Nutr Biochem 2004; 15: 461-466

44 Andreadou I, Iliodromitis EK, Mikros E, Constantinou M, Agalias A, Magiatis P, Skaltsounis AL, Kamber E, Tsantili-Kakoulidou A, Kremastinos DT. The olive constituent oleuropein exhibits anti-Ischemic, antioxidative, and hypolipidemic effects in anesthetized rabbits. J Nutr 2006; 136: 2213-2219

45 Andreadou I, Sigala F, Iliodromitis EK, Papaefthimiou M, Sigalas C, Aligiannis $N$, Savvari P, Gorgoulis V, Papalabros E, Kremastinos DT. Acute doxorubicin cardiotoxicity is successfully treated with the phytochem- ical oleuropein through suppression of oxidative and nitrosative stress. J Mol Cell Cardiol 2007; 42: 549-558

46 Andreadou I, Mikros E, Ioannidis K, Sigala F, Naka K, Kostidis S, Farmakis D, Tenta R, Kavantzas N, Bibli SI, Gikas E, Skaltsounis L, Kremastinos DT, Iliodromitis EK. Oleuropein prevents doxorubicin-induced cardiomyopathy interfering with signaling molecules and cardiomyocyte metabolism. J Mol Cell Cardiol 2014; 69: 4-16

47 Andreadou I, Papaefthimiou M, Zira A, Constantinou M, Sigala F, Skaltsounis AL, Tsantili-Kakoulidou A, Iliodromitis EK, Kremastinos DT, Mikros E. Metabonomic identification of novel biomarkers in doxorubicin cardiotoxicity and protective effect of the natural anti-oxidant oleuropein. NMR Biomed 2009; 22: 585-592

48 Masella R, Varı R, D'Archivio M, Di Benedetto R. Extra virgin olive oil biophenols inhibit cell-mediated oxidation of LDL by increasing the mRNA transcription of glutathione-related enzymes. J Nutr 2004; 134: 785791

49 Coni E, Di Benedetto R, Di Pasquale M, Masella R, Modesti D, Mattei R, Carlini EA. Protective effect of oleuropein, an olive oil biophenol, on low density lipoprotein oxidizability in rabbits. Lipids 2000; 35: 45-54

50 Dell'Agli M, Maschi O, Galli GV, Fagnani R, Dal Cero E, Caruso D, Bosisio E. Inhibition of platelet aggregation by olive oil phenols via cAMP-phosphodiesterase. Br J Nutr 2008; 99: 945-951

51 Masella R, Cantafora A, Modesti D, Cardilli A, Gennaro L, Bocca A, Coni E. Anti-oxidant activity of 3,4-DHPEA-EA and protocatechuic acid: a comparative assessment with other olive oil biophenols. Redox Rep 1999; 4: 113-121

52 Gonzalez-Santiago M, Martin-Bautista E, Carrero JJ, Fonolla J, Baro L, Bortolome MV, Gil-Loyzaga P, Lopez-Huertas E. One month administration of hydroxytyrosol, a phenolic anti-oxidant present in olive oil, to hyperlipemic rabbits inproves blood lipid profile, anti-oxidant status and reduces atherosclerosis development. Atherosclerosis 2006; 188: 35-42

53 O'Dowd Y, Driss F, Dang PM, Elbim C, Gougerot-Pocidalo MA, Pasquier C, El-Benna J. Anti-oxidant effect of hydroxytyrosol, a polyphenol from olive oil: scavenging of hydrogen peroxide but not superoxide anion produced by human neutrophils. Biochem Pharmacol 2004; 68: 2003-2008

54 Rafehi H, Smith AJ, Balcerczyk A, Ziemann M, Ooi J, Loveridge SJ, Baker EK, El-Osta A, Karagiannis TC. Investigation into the biological properties of the olive polyphenol, hydroxytyrosol: mechanistic insights by genome-wide mRNA-Seq analysis. Genes Nutr 2012; 7: 343-355

55 Zrelli H, Matsuoka M, Kitazaki S, Zarrouk M, Miyazaki H. Hydroxytyrosol reduces intracellular reactive oxygen species levels in vascular endothelial cells by upregulating catalase expression through the AMPKFOXO3a pathway. Eur J Pharmacol 2011; 660: 275-282

56 Granados-Principal S, El-Azem N, Pamplona R, Ramirez-Tortosa C, Pulido-Moran M, Vera-Ramirez L, Quiles JL, Sanchez-Rovira P, Naudí A, Portero-Otin M, Perez-Lopez P, Ramirez-Tortosa M. Hydroxytyrosol ameliorates oxidative stress and mitochondrial dysfunction in doxorubicininduced cardiotoxicity in rats with breast cancer. Biochem Pharmacol 2014; 90: 25-33

57 Salami M, Galli C, De Angelis L, Visioli F. Formation of F2-isoprostanes in oxidized low density lipoprotein: inhibitory effect of hydroxytyrosol. Pharmacol Res 1995; 31: 275-279

58 Andreadou I, Farmakis D, Prokovas E, Sigala F, Zoga A, Spyridaki K, Papalois A, Papapetropoulos A, Anastasiou-Nana M, Kremastinos DT, Iliodromitis EK. Short-term statin administration in hypercholesterolaemic rabbits resistant to postconditioning: effects on infarct size, endothelial nitric oxide synthase, and nitro-oxidative stress. Cardiovasc Res 2012; 94: 501-509

59 Schmitt CA, Handler N, Heiss EH, Erker T, Dirsch VM. No evidence for modulation of endothelial nitric oxide synthase by the olive oil polyphenol hydroxytyrosol in human endothelial cells. Atherosclerosis 2007; 195: e58-e64

60 Carluccio MA, Siculella L, Ancora MA, Massaro M, Scoditti E, Storelli C, Visioli F, Distante A, De Caterina R. Olive oil and red wine anti-oxidant polyphenols inhibit endothelial activation: antiatherogenic properties of Mediterranean diet phytochemicals. Arterioscler Thromb Vasc Biol 2003; 23: 622-629

61 Acin S, Navarro MA, Arbones-Mainar JM, Guillen N, Sarria AJ, Carnicer R, Surra JC, Orman I, Segovia JC, Torre Rde L, Covas MI, Fernandez-Bolanos J, Ruiz-Gutierrez V, Osada J. Hydroxytyrosol administration enhances atherosclerotic lesion development in apo E deficient mice. J Biochem 2006; 140: 383-391 
62 Pan S, Liu L, Pan H, Ma Y, Wang D, Kang K, Wang J, Sun B, Sun X, Jiang H. Protective effects of hydroxytyrosol on liver ischemia/reperfusion injury in mice. Mol Nutr Food Res 2013; 57: 1218-1227

63 Czerwińska M, Kiss AK, Naruszewicz M. A comparison of anti-oxidant activities of oleuropein and its dialdehydic derivative from olive oil, oleacein. Food Chem 2012; 131: 940-947

64 Morello JR, Vuorela S, Romero MP, Motilva MJ, Heinonen M. Anti-oxidant activity of olive pulp and olive oil phenolic compounds of the arbequina cultivar. J Agric Food Chem 2005; 53: 2002-2008

65 Rosignoli P, Fuccelli R, Fabiani R, Servili M, Morozzi G. Effect of olive oil phenols on the production of inflammatory mediators in freshly isolated human monocytes. J Nutr Biochem 2013; 24: 1513-1519

66 Angelino D, Gennari L, Blasa M, Selvaggini R, Urbani S, Esposto S, Servili $M$, Ninfali P. Chemical and cellular anti-oxidant activity of phytochemicals purified from olive mill waste waters. J Agric Food Chem 2011; 59: 2011-2018

67 Paiva-Martins F, Fernandes J, Rocha S, Nascimento H, Vitorino R, Amado F, Borges F, Belo L, Santos-Silva A. Effects of olive oil polyphenols on erythrocyte oxidative damage. Mol Nutr Food Res 2009; 53: 609-616

68 Vougogiannopoulou K, Lemus C, Halabalaki M, Pergola C, Werz O, Smith $A B$, Michel S, Skaltsounis L, Deguin B. One-step semisynthesis of oleacein and the determination as a 5-lipoxygenase inhibitor. J Nat Prod 2014; 77: 441-445

69 Czerwinska ME, Kiss AK, Naruszewicz M. Inhibition of human neutrophils NEP activity, CD11b/CD18 expression and elastase release by 3,4-dihydroxyphenylethanol-elenolic acid dialdehyde, oleacein. Food Chem 2014; 153: $1-8$

70 Sindona G, Caruso A, Cozza A, Fiorentini S, Lorusso B, Marini E, Nardi M, Procopio A, Zicari S. Anti-inflammatory effect of 3,4-DHPEA-EDA [2(3,4-hydroxyphenyl) ethyl (3S,4E)-4-formyl-3-(2-oxoethyl)hex-4enoate] on primary human vascular endothelial cells. Curr Med Chem 2012; 19: 4006-4013

71 Di Benedetto R, Varì R, Scazzocchio B, Filesi C, Santangelo C, Giovannini C, Matarrese P, D'Archivio M, Masella R. Tyrosol, the major extra virgin olive oil compound, restored intracellular anti-oxidant defences in spite of its weak antioxidative effectiveness. Nutr Metab Cardiovasc Dis 2007; 17: 535-545

72 Cañuelo A, Peragón J. Proteomics analysis in Caenorhabditis elegans to elucidate the response induced by tyrosol, an olive phenol that stimulates longevity and stress resistance. Proteomics 2013; 13: 3064-3075

73 Vivancos M, Moreno JJ. Effect of resveratrol, tyrosol and beta-sitosterol on oxidised low-density lipoprotein-stimulated oxidative stress, arachidonic acid release and prostaglandin E2 synthesis by RAW 264.7 macrophages. Br J Nutr 2008; 99: 1199-1207

74 Lamuela-Raventos RM, Gimeno E, Fito M, Castellote AI, Covas M, de la Torre-Boronat MC, Lopez-Sabater MC. Interaction of olive oil phenol anti-oxidant components with low-density lipoprotein. Biol Res 2004; 37: $247-252$

75 Samuel SM, Thirunavukkarasu M, Penumathsa SV, Paul D, Maulik N. Akt/FOXO3a/SIRT1 mediated cardioprotection by n-tyrosol against ischemic stress in rat in vivo model of myocardial infarction: switching gears towards survival and longevity. J Agric Food Chem 2008; 56: 9692-9698

76 Thirunavukkarasu M, Mathews Samuel S, Penumathsa SV, Zhan L, Bertelli AAE, Maulik N. Pharmacological preconditioning with tyrosol, a white wine component, depends on the activation of PI-3 kinase pathway in ischemic reperfused rat myocardium. FASEB J 2008; 22: 705.1

77 Sun L, Isaak CK, Zhou Y, Petkau JC, O K, Liu Y, Siow YL. Salidroside and tyrosol from Rhodiola protect H9c2 cells from ischemia/reperfusioninduced apoptosis. Life Sci 2012; 91: 151-158

78 Bali EB, Ergin V, Rackova L, Bayraktar O, Küçükboyaci N, Karasu C. Olive leaf extracts protect cardiomyocytes against 4-hydroxynonenal-induced toxicity in vitro: comparison with oleuropein, hydroxytyrosol, and quercetin. Planta Med 2014; 80: 984-992

79 Paiva-Martins F, Fernandes J, Santos V, Silva L, Borges F, Rocha S, Belo L, Santos-Silva A. Powerful protective role of 3,4-dihydroxyphenylethanol-elenolic acid dialdehyde against erythrocyte oxidative-induced hemolysis. J Agric Food Chem 2010; 58: 135-140

80 Rietjens SJ, Bast A, de Vente J, Haenen GR. The olive oil anti-oxidant hydroxytyrosol efficiently protects against the oxidative stress-induced impairment of the NObullet response of isolated rat aorta. Am J Physiol Heart Circ Physiol 2007; 292: H1931-H1936

81 Fabiani R, Rosignoli P, De Bartolomeo A, Fuccelli R, Servili M, Montedoro GF, Morozzi G. Oxidative DNA damage is prevented by extracts of olive oil, hydroxytyrosol, and other olive phenolic compounds in human blood mononuclear cells and HL60 cells. J Nutr 2008; 138: 1411-1416

82 Parzonko A, Czerwinska ME, Kiss AK, Naruszewicz M. Oleuropein and oleacein may restore biological functions of endothelial progenitor cells impaired by angiotensin II via activation of Nrf2/heme oxygenase-1 pathway. Phytomedicine 2013; 20: 1088-1094

83 Bayram B, Ozcelik B, Grimm S, Roeder T, Schrader C, Ernst IMA, Wagner $A E$, Grune T, Frank J, Rimbach G. A diet rich in olive oil phenolics reduces oxidative stress in the heart of SAMP8 mice by induction of Nrf2-dependent gene expression. Rejuvenation Res 2012; 15: 71-81

84 Fitó M, Covas MI, Lamuela-Raventósc RM, Vilaa J. Protective effect of olive oil and its phenolic compounds against low density lipoprotein oxidation. Lipids 2000; 35: 633-638

85 Poudyal H, Campbell F, Brown L. Olive leaf extract attenuates cardiac, hepatic, and metabolic changes in high carbohydrate-, high fat-fed rats. J Nutr 2010; 140: 946-953

86 Wang L, Geng C, Jiang L, Gong D, Liu D, Yoshimura H, Zhong $L$. The antiatherosclerotic effect of olive leaf extract is related to suppressed inflammatory response in rabbits with experimental atherosclerosis. Eur J Nutr 2008; 47: 235-243

87 Ahmadvand H, Bagheri S, Khosrobeigi A, Boshtam M, Abdolahpour F. Effects of olive leaves extract on LDL oxidation induced-CuSO(4) in vitro. Pak J Pharm Sci 2012; 25: 571-575

88 Singh I, Mok M, Christensen AM, Turner AH, Hawley JA. The effects of polyphenols in olive leaves on platelet function. Nutr Metab Cardiovasc Dis 2008; 18: 127-132 